\title{
Inference in cognitive maps
}

\author{
Michael P. Wellman \\ University of Michigan, Artificial Intelligence Laboratory, 1101 Beal Avenue, Ann Arbor, MI 48109, USA
}

\begin{abstract}
Cognitive mapping is a qualitative decision modeling technique developed over twenty years ago by political scientists, which continues to see occasional use in social science and decision-aiding applications. In this paper, I show how cognitive maps can be viewed in the context of more recent formalisms for qualitative decision modeling, and how the latter provide a firm semantic foundation that can facilitate the development of more powerful inference procedures as well as extensions in expressiveness for models of this sort.
\end{abstract}

\section{Introduction}

The term cognitive map as used here refers to a particular form of graphical, qualitative model of a decision maker's subjective beliefs. ${ }^{1}$ The distinguishing characteristic of these models is a focus on abstract causal relationships among concepts, specifically on the directions, or signs, of causal dependence. Cognitive maps attempt to capture the qualitative structure of a belief system as it relates to a decision problem, by representing the directional relationships among component beliefs.

Cognitive maps were introduced by political scientists in the early 1970s as a formal tool for the analysis of policymaking behavior [1]. The motivations for focusing on qualitative, or sign, relationships were twofold:

(1) The direction of relationships is typically all that is discernible from transcripts of policy sessions or verbal arguments.

(2) The social scientists' primary interest is in capturing the structure of a decision problem and determining the relationship among arguments, as opposed to prescribing a policy alternative.

\footnotetext{
$\overline{1}$ An alternate, common, but completely independent usage applies to a type of model of geographical knowledge originally developed by Lynch [17].
} 
In the ensuing years, political scientists generated cognitive maps as part of several historical studies $[1,2,4,16]$. Advocates of the technique claimed that the models faithfully captured the structure of arguments and offered some insights into policy practice. Moreover, the maps were simple to analyze, and although constructing them from raw historical data was labor-intensive, the process was amenable to robust modeling methodology. Nevertheless, their use does not seem to be very widespread in political science today, perhaps due largely to the difficulties of encoding. ${ }^{2}$ However, others continue to apply cognitive maps and similar formalisms, notably to analyze the belief structures of business decision makers $[3,13]$.

Cognitive mapping as practiced in political science and organization theory is primarily for descriptive purposes. Decision consultants have also employed cognitive maps prescriptively: constructing maps modeling individual and group perspectives on organizational problems, and then using these models to facilitate communication and decision making [11]. ${ }^{3}$ Cognitive maps have also been used to support knowledge acquisition, specifically in devices for structuring the task of quantitative modeling performed by human decision analysts [6].

Cognitive maps are interesting to us in part for their potential practical relevance to social science and cognitive modeling. Successful application in those areas would enhance their utility for decision support, as it could provide a bridge to other descriptions of subjective beliefs and preferences. Methodologies designed to elicit cognitive maps via interviews or from textual records could be adapted to decision-support contexts. (But ultimately, their usefulness as a foundation for decision analysis does not absolutely depend on cognitive validity.) In addition, cognitive maps possess historical interest as a relatively early (compared to qualitative reasoning in $\mathrm{AI}$ ) attempt to formalize a form of qualitative reasoning in a decision-making context.

In previous work, I have developed a formalism for qualitative probabilistic reasoning with some significant similarities to cognitive maps. ${ }^{4}$ I have done so with the benefit of several technical innovations not available to the originators of cognitive mapping. In particular, the advent of graphical dependency models for probabilistic reasoning [19] and of sign algebras for qualitative reasoning [22], both in AI, can substantially solidify the technical underpinnings of cognitive maps. In doing so, we can both extend the formalism and improve our understanding of the assumptions and implications of alternate modeling choices and analysis procedures.

\section{Cognitive maps}

Technically, cognitive maps are directed graphs with signed edges. In formal notation, a cognitive map $M$ is a pair, $M=(C, E)$, where $C$ is a set of nodes, or concepts, and $E$ is a set of

\footnotetext{
$\overline{2}$ Robert Axelrod, personal communication.

3 It is unclear to me whether this line of research on cognitive maps derives historically from the work in political science. Rather, Eden [10] stresses its antecedents in Kelly's Personal Construct Theory [14], viewing cognitive maps as an abstraction and structurally explicit version of the Repertory Grid. He credits Tolman [21] with originating the term "cognitive map", but Tolman's work is clearly concerned with geographical maps, not (except analogically) causal graphs of general belief structures.

${ }^{4}$ The existence of cognitive maps and their relationship to my work on qualitative probabilistic reasoning [24] was brought to my attention by Dennis Buede in 1991 .
} 
signed edges,

$$
E=\left\{\left(c, c^{\prime}, \delta\right): c, c^{\prime} \in C, \text { there is an edge of sign } \delta \text { from } c \text { to } c^{\prime}\right\}
$$

The possible signs $\delta$ are usually + and -, although sometimes there are strict and non-strict versions, sometimes relations of sign 0 are made explicit, and ambiguous relations of sign "?" are sometimes allowed. That seems to be the extent of the mathematical structure we can attribute to cognitive maps in general. Further restrictions, such as acyclicity, may be imposed in particular cases.

The signed edges are intended to express the positivity or negativity of the causal relation between the connected concepts. For example, the cognitive map of Fig. 1, taken from Levi and Tetlock's study of the Japanese decision to attack Pearl Harbor [16], represents the causal argument that remaining idle would cause attrition of Japanese strength while enhancing the defensive preparedness of the US, both of which decrease Japanese prospects for success in war. Note that the concepts' domains are not necessarily defined precisely. For example, remaining idle might be a binary proposition or may denote the amount of time of idleness. Similarly, there are no obvious scales for measuring Japanese strength, US preparedness, or success in war. Nevertheless, it seems easy to grasp the intended meaning of the signed relationships in this model.

The intended inferences to draw from this cognitive map are just the obvious ones. Remaining idle decreases the prospects for Japanese success in war along two causal paths. Since both paths agree, the relationship between idleness and war prospects is negative. So given a belief that war is inevitable, starting it sooner would be better.

Of course, cognitive maps may be more complex than that depicted in Figure 1 (see the references for larger examples).

The general definition of the derived relationship between two concepts in an acyclic cognitive map is simply the sum of the signs for all paths between them, where the sign of a path is the product of the signs of its constituent links. ${ }^{5}$ Let $P_{a, b}$ be the set of paths in $M$ from $a$ to $b$. Then the sign of the derived causal relation from $a$ to $b$ is [1]:

$$
\bigoplus_{p \in P_{a, b}}\left(\underset{\left(c, c^{\prime}, \delta\right) \in p}{\otimes} \delta\right),
$$

where $\oplus$ and $\otimes$ denote sign addition and multiplication, respectively. In other words, the derived relation between $a$ and $b$ is the sign sum of the relations along all paths, where the relation on a path is the sign product of the relations on its constituent edges. Although $P_{a, b}$

\footnotetext{
5 The situation is more complicated for cognitive maps with cycles. Axelrod [1] suggest that one add the signs of all (simple) paths and cycles. But it is not clear which cycles are to be included when querying the relation between two concepts, and also appears questionable whether a negative feedback path should contribute to a negative overall relation. Maps with feedback may perhaps be more useful for studying issues such as stability, which is sometimes determined by signs alone. As we are interested primarily in derived relations, for the subsequent discussion we assume acyclic maps.
} 


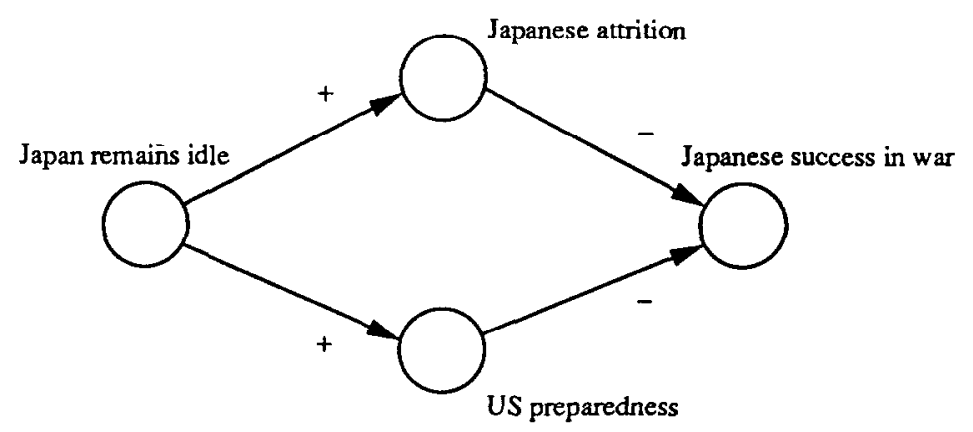

Fig. 1. A simple cognitive map (from Levi and Tetlock [16]).

may contain an exponential number of paths, the value of (1) can be computed in time $O(|E|)$ using standard graph-labeling techniques.

\section{Semantics}

\subsection{Motivation}

Although the results from path analysis of a cognitive map may seem intuitive, in order to judge whether such conclusions are valid we require a more precise account of the meaning of the primitive concepts. Computing the derived relation via (1) is a form of inference: from the signs of certain links and the absence of others we conclude the derived sign of an arbitrary concept pair. In applying path analysis or any other inference procedure to cognitive maps, we would like some assurance that it is sound, and perhaps would be interested to know whether it is complete.

But is not the soundness of path tracing obvious? Obviousness is in the mind of the intuiter, of course, but some subtle problems arise for some seemingly reasonable interpretations of concepts and signed relations. For example, if the signs denote positive or negative correlation and the concepts random variables, then path tracing is not sound. If $x$ is positively correlated with $y$, and $y$ positively correlated with $z$, it is still quite possible that $x$ and $z$ be negatively correlated, even if they are conditionally independent given $y$. Thus, correlation is evidently not a good interpretation for the sign of relationships.

The case for a firm semantics is even stronger when we consider more complicated forms of inference. For example, we might be interested in deriving evidential relations among the concepts, that is, those going against the causal direction of the cognitive map. For example, historians might ask whether, according to the model of Fig. 1, the fact that the Japanese lost the war is evidence that they waited too long. Or perhaps we are interested in determining relations among effects of the same cause (Japanese attrition and US preparedness), or causes of the same effect (attrition and preparedness again). This latter form of relation is called intercausal and is an important component of diagnosis and explanation, which I return to below. And in any of these cases, we might want to conditionalize our conclusion on partial 
information about the concepts, assuming that the values of some of them may have been observed or otherwise revealed.

Finally, a precise description of the meaning of these basic qualitative relations is indispensable if we wish to extend the formalism to include new qualitative relations. In order to augment cognitive maps to incorporate higher-order relations, functional dependencies, some numeric or algebraic information, or any other type of constraint, we need to understand how the new relation type complements, subsumes, or otherwise interacts with the basic sign relations.

\subsection{Abstract causal relations}

What form should a semantics for cognitive maps take? To serve the objectives described above, our semantics should provide a definition for abstract causal (sign) relations so that we can determine the validity of inference rules such as (1). The definition should be local as far as possible, so that in assessing the validity of a signed edge $\left(c, c^{\prime}, \delta\right)$, we can limit our attention to a neighborhood of concepts $c$ and $c^{\prime}$. It should be unambiguously determined by the precise causal relation among the concepts, so that the sign relation is an abstraction of the precise relation. If the precise relation is a functional dependency, the sign would be an abstraction of the function relating the concepts. If the causal relation is probabilistic, the sign would be an abstraction of the probabilistic dependence, defined in terms of conditional probability. Since the probabilistic case is more general (and many of the uses of cognitive maps involve causal rclations with considerable uncertainty), we focus on a semantics of abstract probabilistic dependencies. ${ }^{6}$

\section{Qualitative probabilistic networks}

In previous work, I have developed a formalism, called qualitative probabilistic networks (QPNs), which shares some significant features with cognitive maps. QPNs have been described in detail elsewhere [24]; this paper mentions only some of the salient aspects. Like cognitive maps, QPNs are signed directed acyclic ${ }^{7}$ graphs, with nodes denoting concepts and signed edges denoting abstract causal relations.

Concepts are interpreted as random variables, although the variables' domains need not be explicitly specified. ${ }^{8}$ In this respect QPNs resemble cognitive maps, where we can express concepts such as "Japanese attrition" without identifying a measurement scale. In some descriptions of cognitive maps (e.g., Eden [10]), variables are defined in terms of their endpoints, or "poles"; semantically, however, what matters is the relative ordering among values, not whether they are at the extremes.

\footnotetext{
${ }^{6}$ For details on how to take advantage of the stronger constraints inherent in qualitative functional dependencies, when applicable, see [25].

${ }^{7}$ As mentioned above, cognitive maps as originally defined need not be acyclic, but the path analysis techniques are uncontroversially applicable only in the acyclic case.

${ }^{8}$ This is a fine point: the semantic interpretation refers to values in the domain of the variahle, but uses of QPNs need not specify the values.
} 
Edges in a QPN denote the sign of probabilistic dependence. Specifically, an edge $\left(c, c^{\prime},+\right)$ in a QPN $G$ means that, for all values $c_{1}>c_{2}$ of $c, c_{0}^{\prime}$ of $c^{\prime}$, and all assignments $x$ to other predecessors of $c^{\prime}$ in $G$,

$$
\operatorname{Pr}\left(c^{\prime} \geqslant c_{0}^{\prime} \mid c_{1} x\right) \geqslant \operatorname{Pr}\left(c^{\prime} \geqslant c_{0}^{\prime} \mid c_{2} x\right) .
$$

An edge $\left(c, c^{\prime},-\right)$ is defined analogously, with $\leq$ substituted for the central inequality in (2). If there is no edge from $c$ to $c^{\prime}$ (or, equivalently, an edge of sign 0 ), and no path from $c^{\prime}$ to $c$ (otherwise, $\left(c, c^{\prime}, \delta\right)$ would create a cycle), then the left- and right-hand sides of (2) are equal. If none of these cases hold, and there is no path from $c^{\prime}$ to $c$, then there is an ambiguous edge $\left(c, c^{\prime}, ?\right)$.

Note that when (2) holds with equality (the edge has sign 0 , or is absent), then $c$ and $c^{\prime}$ are conditionally independent given the predecessors of $c^{\prime}$. The independence properties of QPNs are identical to those of numeric probabilistic dependency networks [19], so all results and algorithms based on graph structure alone carry over.

There are two compelling reasons to adopt this definition for signed edges in cognitive maps as well. First, inference via path analysis - formula (1) - is valid under this definition, as long as $a$ and $b$ have no common ancestors. ${ }^{9}$ Second, among a broad class of potential definitions (those based on relative conditional probabilities of the effect, given various possibilities for the cause; see [24] for details), this is the only one for which path analysis is valid. We have already noted above that a definition based on correlation, for example, would not suffice. Since path analysis scems a minimal requircment for an intuitive, nontrivial inference mechanism, the case for this particular semantic account is quite strong.

\section{Non-causal paths}

The path analysis formula (1) applies only to directed paths from $a$ to $b$. This corresponds to pure causal inference, the situation depicted in Fig. 2(a). However, in a cognitive map, there may be many undirected pathways among a pair of variables, only some of which are purely causal paths. With the probabilistic definition above, we can consider the implications of non-causal pathways on the results of path analysis. We can also address the issue of instantiated evidence, that is, situations where the values of one or more variables have been observed.

For instance, if a variable on a causal pathway has been observed, it has the effect of blocking that path. If $e$ is observed evidence, then in asking for the derived causal relation of $a$ on $b$ given $e$, we should ignore (remove from $P_{a, b}$ ) any paths that - like that depicted in Fig. 2(b) - traverse $e$. This follows from the conditional independence properties of probabilistic networks.

\footnotetext{
${ }^{9}$ The original path analysis definition ignores the possibility of common ancestors, which does not seem justifiable. For a proof of the validity of the basic chaining operation, see [241. If $a$ and $b$ have no common ancestors, repeated application of this operation is sufficient.
} 
(a)

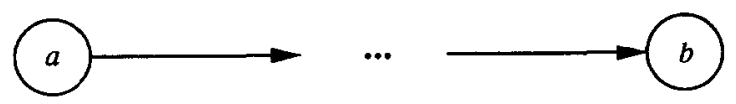

(b)

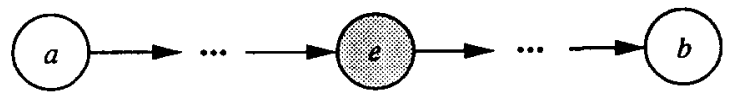

(c)

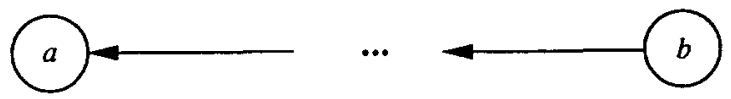

(d)

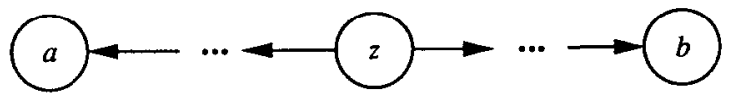

(e)

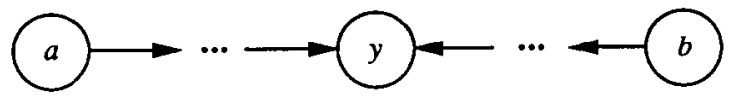

Fig. 2. Patterns of inference from $a$ to $b$ : (a) pure causal inference, (b) causal pathway blocked by evidence, $e$, (c) evidential inference, (d) effects with a common causal ancestor, $z$ (e) causes with a common effect, $y$.

Next, let us consider inference on pathways that cut against the causal grain. Inference from effect to cause is called evidential reasoning, and is common in diagnosis and other reasoning tasks. It is easy to show by applying Bayes's rule to (2) that the sign of probabilistic dependence from $c^{\prime}$ to $c$ is the same as that from $c$ to $c^{\prime}$, and hence when $b$ is a causal ancestor of $a$ (as in Fig. 2(c)), the same formula for path analysis applies (note that the exclusion of cycles implies that we need only perform path analysis one way or the other).

In the case (Fig. 2(d)) where $a$ and $b$ have a common ancestor, $z$, there exists an evidential path from $a$ to $z$, as well as causal path from $z$ to $b$. By the previous argument, the evidential path can be treated just like the causal path, with the sign from $a$ to $z$ behaving as if the path were in the reverse direction. This can then be combined with the causal path from $z$ to $b$, yielding a total derived relation along this mixed path from $a$ to $z$ to $b$ equal to the sign multiplication of all its edges. As Druzdzel and Henrion have recently shown [7,8], the signs along all paths can be considered independently, and thus we can simply include mixed paths consisting of an evidential followed by a causal subpath in the set $P_{a, b}$ of formula (1).

Finally, we must consider the case (Fig. 2(e)) where an undirected pathway between $a$ and $b$ includes a concept $y$ with both edges leading in. For example, $a$ and $b$ might have a common descendant. These pathways can be ignored, unless $y$ or one of its descendants has been observed. In that case, the parents of $y$ are related by an intercausal relation. This means that given $y$, knowing whether one of $y$ 's potential causes obtained gives us information about the other potential cause. The sign of the intercausal relation cannot be determined by the abstract causal relations in basic cognitive maps, however. 


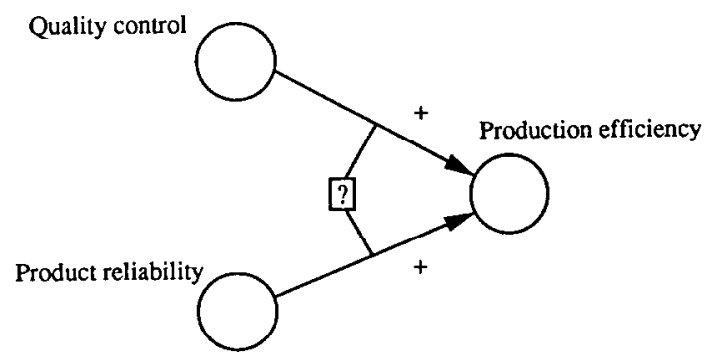

Fig. 3. A cognitive map fragment (from Narayanan and Fahey [18]) with intercausal interactions.

\section{Intercausal reasoning}

In comparison with causal and evidential inference, intercausal reasoning has received relatively little attention. Nevertheless, formation of derived relations among causes of observed effects is ubiquitous in diagnosis, argumentation, and explanation. By examining the semantics of joint causal relations, we can determine conditions under which such inferences are warranted.

\subsection{Qualitative synergy}

The relation between two causes given their common effect depends on how they interact in producing the effect. If two causes are competing, then establishing one would tend to decrease belief in the other. This phenomenon is called explaining away [19]. If they are complementary, then the opposite phenomenon would obtain. For example, in the fragment of Fig. 3, quality control and product reliability are two causal influences on production efficiency. Instituting a quality control rcgime and improving the design of the product are two ways of improving efficiency, so if we observe that a company has increased productivity, we might hypothesize that they have taken one or both of these measures. But once we find out for certain that they have taken one of them (say quality control), this would tend to decrease our degree of belief in the hypothesis that they have taken the other (product redesign), since the former is sufficient to "explain away" the observation. However, it is also plausible that these measures are highly complementary, for example if the product redesign is geared specifically to facilitate quality control. In that case, an increase in belief in one hypothesis would tend to reinforce the other.

A precise probabilistic condition distinguishing these cases, called product synergy, has a form similar to that of our basic abstract causal relation (2):

Definition 1 (product synergy [26]). Let $c$ and $c^{\prime}$ be predecessors of $y$, and let $x$ denote an assignment to $y$ 's other predecessors, if any. Variables $c$ and $c^{\prime}$ exhibit negative product synergy with respect to a particular value $y_{0}$ of $y$, if, for all $c_{1}>c_{2}, c_{1}^{\prime}>c_{2}^{\prime}$, and $x$,

$$
\operatorname{Pr}\left(y=y_{0} \mid c_{1} c_{1}^{\prime} x\right) \operatorname{Pr}\left(y=y_{0} \mid c_{2} c_{2}^{\prime} x\right) \leqslant \operatorname{Pr}\left(y=y_{0} \mid c_{1} c_{2}^{\prime} x\right) \operatorname{Pr}\left(y=y_{0} \mid c_{2} c_{1}^{\prime} x\right) \text {. }
$$

Positive and zero product synergy are defined analogously. The sign of product synergy corresponds directly to the sign of the intercausal relation of $c$ on $c^{\prime}$ given an observation $y_{0}$.

We can extend cognitive maps to perform intercausal inference as well as the causal and evidential sort by augmenting the causal relations to include product synergy. Then, the path 
analysis procedure can be extended to account for pathways with instantiated evidence, that is, the situation of Fig. 2(e) where $y$ or some descendant of $y$ denotes observed evidence. Efficient algorithms based on propagation of signs along the graph have been described by Druzdzel and Henrion [8]. For the gencral case where there may be undirected loops in the graph (or we wish to average over unobserved causal variables not participating in the intercausal pathway), we need to strengthen (3) to hold with respect to any probability distribution over $x$ [9].

\subsection{Qualitative explanation}

One prominent application of cognitive maps was to the study of political argumentation [2]. If the cognitive map represents the belief structure of a political agent, then we should be able to apply typical operations on arguments to the map structure. For example, a common tactic in argument is to posit facts or pose hypotheticals, examining the implications derived from the belief structure conditional on the posited fact or hypothetical. This is a form of explanation problem: given some evidence, describe how the belief structure would explain the evidence and what further conclusions would be drawn.

Intercausal inference plays a significant role in qualitative explanation [12]. In describing how beliefs would be modified by evidence, one needs to establish whether arguments compete and explain away each other, or whether they are complementary and support each other.

\section{Decision making}

In most of the cognitive maps developed by political scientists, causal paths begin with policy variables, and end with variables representing some stakeholders' utility. Thus, overall arguments are of the form, "this policy option will be beneficial (deleterious) to that group". Presumably, the map endorses an option if it can be shown to qualitatively increase the utility of all the groups of interest. The power to prescribe decisions can also be justified by an appeal to qualitative probabilistic networks.

In QPNs (as in influence diagrams [20]), we distinguish decision variables under the agent's direct control, and designate one variable as the value node denoting the objective. Edges into the value node describe the qualitative functional relation of the antecedent concept on utility. Derived relations on utility can be computed using the same path analysis routines that apply to random variables. As expected, if the policy variable has an unambiguous sign with respect to utility, then the recommended policy option is determined.

In addition, we can often derive policy implications even when the absolute relations of policy variables to utility are ambiguous. To do so, we require another qualitative synergy concept describing the interactions among causal variables on other random variables or on utility.

Definition 2 (additive synergy [24]). Let $c$ and $c^{\prime}$ be predecessors of $y$, and let $x$ denote an assignment to $y$ 's other predecessors, if any. Variables $c$ and $c^{\prime}$ exhibit positive product synergy with respect to $y$, if, for all $c_{1}>c_{2}, c_{1}^{\prime}>c_{2}^{\prime}, y_{0}$, and $x$,

$$
\operatorname{Pr}\left(y \geqslant y_{0} \mid c_{1} c_{1}^{\prime} x\right)+\operatorname{Pr}\left(y \geqslant y_{0} \mid c_{2} c_{2}^{\prime} x\right) \geqslant \operatorname{Pr}\left(y \geqslant y_{0} \mid c_{1} c_{2}^{\prime} x\right)+\operatorname{Pr}\left(y \geqslant y_{0} \mid c_{2} c_{1}^{\prime} x\right) .
$$


Note that, in contrast to product synergy, additive synergy is defined with respect to a variable $y$, not a specific value. The definitions of negative and zero additive synergy are analogous.

If a decision variable $d$ and an observed variable $e$ have a positive synergy on utility, then the optimal policy for $d$ must be monotonically increasing in $e$. Synergy on random variables can also be propagated through qualitative influences to reveal indirect synergy relations (and thereby monotone policy constraints) holding in a complex network. For examples and details, see $[23,24]$.

\section{Other forms of map analysis}

Several forms of analysis that have becn applied to cognitive maps are not supported or even addressed by the probabilistic semantics presented here. For example, several of the studies of cognitive maps of policymakers and business managers draw conclusions based on measures of the complexity of the map structure $[16,18]$ or on its change in structure over time $[3,18]$. For the most part, these complexity and change measures are simply derived from the size of the graph (nodes and links) or number of common components, and do not make any use of the sign relations or more complex argument structure. The validity of such measures with respect to the hypotheses being investigated requires some further assumptions about the meaning of the cognitive maps, but this would seem to go beyond the semantics of individual causal relations.

\section{Related work}

There has been no previous notice, to my knowledge, of cognitive maps by the AI qualitative reasoning community. The only work I am aware of in the uncertain reasoning community directly addressing cognitive maps are some fuzzy extensions [15,27]. However, fuzzy cognitive maps are not really qualitative models at all, but rather quantitative models based on particular techniques for associating numbers or intervals with edges on directed graphs. Like cognitive maps, the quantities combine by propagation along paths, but there is no other connection to the original spirit of cognitive maps. Their interpretation of a sign relation is a fuzzy interval, but this has no semantic account in terms of beliefs or other fundamental relation.

There are other modeling formalisms that resemble cognitive maps in associating signs with edges in a directed graph (for example, some qualitative econometric models). Where the graphs are acyclic and the nodes can be considered random variables, the QPN definitions provide a suitable semantics - and the inference algorithms a computational mechanism for these formalisms as well.

\section{Conclusion}

Cognitive maps were originally designed to capture the intuitive, qualitative, causal relationships thought to represent the structure of a political decision problem. Some simple inference 
mechanisms - based on path analysis from cause to effect - were proposed to draw conclusions about the consequences of a cognitive map. By defining a precise semantic interpretation of qualitative causality in terms of abstracted probabilistic relationships, we can justify these inference mechanisms as well as extend them to address evidential and intercausal inference patterns. Using transformation-based or propagation-based inference procedures, we can derive relations among arbitrarily connected concepts.

In extending our inference to the realm of intercausal reasoning and decision making, we find it necessary and useful to introduce new, higher-order qualitative synergy relations. The new relations correspond to intuitive interaction concepts (competition or complementarily), are robust under natural inference operations (e.g., chaining), and justify useful qualitative conclusions about patterns of reasoning (e.g., explaining away) or optimal decisions (e.g., monotone policies). Undoubtedly there are other critical qualitative distinctions waiting to be identified, enriching our representations and extending the scope and power of our qualitative modeling techniques.

\section{Acknowledgment}

I would like to thank Dennis Bucde, Marck Druzdzcl, Max Henrion, and Shawn O'Donnell for helpful pointers and valuable discussions on this topic.

\section{References}

[1] R. Axelrod, ed., Structure of Decision: The Cognitive Maps of Political Elites (Princeton Univ. Press, Princeton, NJ, 1976).

[2] R. Axelrod, Argumentation in foreign policy settings, J. Conflict Resolution 21 (1977) 727-755.

[3] P.S. Barr, J.L. Stimpert and A.S. Huff, Cognitive change, strategic action, and organizational rencwal, Strategic Management J. 13 (1992) 15-35.

[4] G.M. Bonham and M.J. Shapiro, Mapping structures of thought, in: I.N. Gallhofer, W.E. Saris, and M. Melman, eds., Different Text Analysis Procedures (Sociometric Research Foundation, 1986).

[5] P.P. Bonissone, M. Henrion, L.N. Kanal and J.F. Lemmer, eds., Uncertainty in Artificial Intelligence 6 (North-Holland, Amsterdam, 1991).

[6] D.M. Buede and D.O. Ferrell, Covergence in problem solving: A prelude to quantitative analysis, Technical report, Paradigm Development Group, Ltd, Fairfax, VA, August 1991.

[7] M.J. Druzdzel and M. Henrion, Belief propagation in qualitative probabilistic networks, in: International Workshop on Qualitative Reasoning and Decision Technologies, Barcelona, Spain, IMACS (1993) 451-460.

[8] M.J. Druzdzel and M. Henrion, Efficient reasoning in qualitative probabilistic networks, in: Proceedings of the National Conference on Artificial Intelligence, Washington, DC, AAAI (1993) 548-553.

[9] M.J. Druzdzel and M. Henrion. Intercausal reasoning with uninstantiated ancestor nodes, in: Proceedings of the Ninth Conference on Uncertainty in Artificial Intelligencc, Washington, DC (1993) 317-325.

[10] C. Eden, Cognitive mapping, European J. Oper. Res. 36 (1988) 1-13.

[11] C. Eden, S. Jones, and D. Sims, Messing About in Problems (Pergamon Press, 1983).

[12] M. Henrion and M.J. Druzdzel, Qualitative propagation and scenario-based explanation of probabilistic reasoning, in: Bonissone et al. [5].

[13] A. Sigismund Huff, ed., Mapping Strategic Thought (Wiley, New York, 1990).

[14] G.A. Kelly, The Psychology of Personal Constructs (W.W. Norton, 1955). 
[15] B. Kosko, Fuzzy cognitive maps, Internat. J. Man-Machine Studies 24 (1986) 65-75.

[16] A. Levi and P.E. Tetlock, A cognitive analysis of Japan's 1941 decision for war, J. Conflict Resolution 24 (1980) 195-211.

[17] K. Lynch, The Image of the City (MIT Press, Cambridge, MA, 1960).

[18] V.K. Narayanan and L. Fahey, Evolution of revealed causal maps during decline: A case study of Admiral, in: Huff [13] 109-133.

[19] J. Pearl, Probabilistic Reasoning in Intelligent Systems: Networks of Plausible Inference (Morgan Kaufmann, Los Altos, CA, 1988).

[20] R.D. Shachter, Evaluating influence diagrams, Oper. Res. 34 (1986) 871-882.

[21] E.C. Tolman, Cognitive maps in rats and men, Psychological Review, 55 (1948) 189-208.

[22] D.S. Weld and J. de Kleer, eds., Readings in Qualitative Reasoning About Physical Systems (Morgan Kaufmann, 1989).

[23] M.P. Wellman, Formulation of Tradeoffs in Planning Under Uncertainty (Pitman, London, 1990).

[24] M.P. Wellman, Fundamental concepts of qualitative probabilistic networks, Artificial Intelligence 44 (1990) 257-303.

[25] M.P. Wellman, Exploiting functional dependencies in qualitative probabilistic reasoning, in: Bonissone et al. [5] 3-15.

[26] M.P. Wellman and M. Henrion, Explaining "explaining away", IEEE Trans. Pattern Analysis and Machine Intelligence 15 (1993) 287-292.

[27] W.R. Zhang, S.S. Chen and J.C. Bezdek, Pool2: A generic system for cognitive map development and decision analysis, IEEE Trans. Systems, Man, and Cybernetics 19 (1989) 31-39. 